\title{
Perfil de salud mental en víctimas del desplazamiento forzado con síntomas de TEPT en Barranquilla, Colombia
}

\section{Mental Health Profile in Victims of Forced Displacement With Symptoms of PTSD in Barranquilla, Colombia}

\author{
Diana Paola Mayor Molinares, Alberto Mario De Castro Correa, \\ María del Carmen Amarís Macías y Fabián David Buelvas González \\ Universidad del Norte, Colombia.
}

(Rec: abril de 2018 - Acept: noviembre de 2018)

\begin{abstract}
Resumen
Se describe el perfil de salud mental en víctimas del desplazamiento forzado con síntomas de TEPT de la urbanización Villas de San Pablo, en Barranquilla (Colombia). La investigación se realizó a partir de exploraciones en entrevistas a profundidad, taller investigativo, y un registro observacional con indicadores, creados a partir de las dimensiones del bienestar psicológico de Ryff, subjetivo de Diener, social de Keyes, y estrategias de afrontamiento de la Escala de Estrategias de Coping - Modificada (EEC-M). Las categorías derivadas del análisis de contenido permitieron identificar los recursos psicosociales con los que cuentan los individuos. Estos recursos fueron englobados en factores protectores y de riesgo, evidenciando estilos de afrontamiento activos y evitativos/pasivos que revelaron un perfil de salud mental semejante a los descritos en la literatura sobre víctimas de violencia socio-política. Se incita a crear programas que aporten a la salud mental de los individuos mediante la promoción de factores protectores.
\end{abstract}

Palabras clave: Desplazamiento forzado; afrontamiento; salud mental; bienestar.

\begin{abstract}
Mental health profile will be described in victims of forced displacement with PTSD symptoms from Villas de San Pablo urbanization, in Barranquilla, Colombia. The study is based on the exploration carried out through an investigative workshop and in-depth interviews, as well as an observatory register by means of indicators, created from the Ryff's psychological well-being dimensions, Diener's subjective well-being, and Keyes's Social Well-Being, and coping strategies from the Coping Strategies Scale-Modified (EEC-M). The categories derived from the content analysis allowed to identify the psychosocial resources, classified as protective or risk factors, displaying actives and evitative/passives coping styles such as showing a mental health profile similar to the victims of socio-political violence literature. It is encouraged to create programs that contribute to the mental health of individuals through the promotion of protective factors.
\end{abstract}

Keywords: Forced displacement; coping; mental health; well-being.

Correspondencia a: Diana Paola Mayor Molinares, Universidad del Norte, Colombia. Dirección: Km 5, Vía Puerto Colombia, Barranquilla, Colombia. E-mail: anaidmar90@gmail.com

Este artículo deriva de la tesis doctoral denominada "Evaluación de un programa de intervención psicosocial para el mejoramiento de la salud mental en víctimas del desplazamiento forzado en Barranquilla, Colombia" respaldado por el Departamento Administrativo de Ciencia, Tecnología e Innovación COLCIENCIAS, COLFUTURO y la Gobernación del Atlántico: Formación de Capital Humano de Alto Nivel para el Departamento del Atlántico, convocatoria No. 673 de 2014. 


\section{Introducción}

\section{El impacto del desplazamiento forzado en las víctimas}

En Colombia, la problemática del conflicto armado interno ha permanecido desde hace más de cinco décadas, impactando en el aspecto económico, social y personal (Tamayo-Agudelo \& Bell, 2018); deteriorando la calidad de vida, y la salud física y mental de la población (Gómez-Restrepo, Tamayo-Martínez, Buitrago, Guarnizo-Herreño, Garzón-Orjuela, Eslava-Schmalbachf, de Vries, Rengifo, Rodríguez y Rincón, 2016); convirtiéndola en un problema de salud pública que produce diferentes formas de violencia sociopolítica (desplazamiento forzado, desapariciones, masacres, torturas, y violencia sexual) (Campo-Arias, Oviedo y Herazo, 2014). La violencia, de la cual deriva el desplazamiento, es definida como un modo de conflicto o medio de lucha social y política, que busca mantener, transformar, cambiar o erradicar un modelo de Estado o afectar a un colectivo humano (esté o no organizado), por su identidad social, étnico-racial, política, gremial, ideológica, religiosa o cultural (Campo-Arias et al., 2014., Arias, 2013). Los impactos creados por este tipo de violencia han generado múltiples emergencias humanitarias las cuales se han incrementado "como resultado de un cambio importante en los patrones de los conflictos armados o guerras en los últimos 50 años, que han cambiado de enfrentamientos entre Estados a enfrentamientos internos" (Arias, 2013, p 278).

En el país, la violencia sociopolítica ha generado un masivo desplazamiento que la ha llevado a tener la "mayor población de desplazados internos afectados por conflictos en el mundo" (Shultz, Gómez, Espinel, Rios, Fonseca \& Hernandez, 2014, p.14), produciendo daños materiales e inmateriales en individuos, familias y comunidades. Los daños materiales causan un daño emergente y gastos económicos, mientras que los inmateriales se relacionan con el sufrimiento moral, el cambio del proyecto de vida, y el deterioro de las relaciones individuales y comunitarias (Campo-Arias et al., 2014), encabezadas por la estigma-discriminación, incrementado en las dos últimas décadas (Campo-Arias y Herazo, 2014).

El Trastorno de Estrés Postraumático (TEPT), es una de las múltiples secuelas de la exposición a la violencia que afecta a las víctimas de desplazamiento forzado. La American Psychiatric Association, Kupfer, Regier, Arango, Ayuso-Mateos, Vieta \& Bagney (2014), definieron al TEPT, en el DSM-5 (Manual Diagnóstico y Estadístico de los Trastornos Mentales) como "el desarrollo de síntomas específicos tras la exposición a uno o más eventos traumáticos" (p. 274). Estudios donde se expone sobre el TEPT en víctimas en Colombia se pueden encontrar en Castaño, Sierra, Sánchez, Torres, Salas y Buitrago (2018), Tamayo-Agudelo y Bell (2018), Hewitt, Juárez, Parada, Guerrero, Romero, Salgado y Vargas (2016), Tamayo, Rincón, De Santacruz, Bautista, Collazos y Gómez-Restrepo (2016), Encuesta Nacional de Salud Mental -ENSM 2015 (Ministerio de Salud y Protección Social, Colciencias, Pontificia Universidad Javeriana, Datos, Procesos y Tecnología SAS, 2015), entre otros.

A pesar de conocer las nefastas consecuencias del desplazamiento en la salud de los colombianos desplazados (como en el caso del TEPT); existen vacíos sobre las investigaciones en salud mental relacionadas con la violencia (Campo-Arias et al., 2014; Aguilar, Morillas y Patró, 2011), así como en estudios que evalúen la efectividad de las intervenciones, presentán- dose, además, problemáticas importantes para abordar las necesidades de salud mental relacionadas con el conflicto armado interno (Tamayo-Agudelo \& Bell, 2018).

En el caso de Barranquilla, se hallan publicaciones que la muestran como una de las principales ciudades receptoras de población afectada por el conflicto armado. Dentro de estas publicaciones se encuentra a Ramos-Vidal (2018), Silvera y Amarís (2017), Valencia (2017). De acuerdo a Ramos-Vidal (2018), los desplazados viven mayoritariamente en zonas de estrato socioeconómico 1 y 2 , implicando un proceso de ajuste en el que la persona desplazada debe ubicarse "en zonas de bajos recursos que habitualmente se sitúan en la periferia de las ciudades" (p. 306). Al ser Barranquilla una de las ciudades principales del Caribe y de Colombia, las víctimas la perciben como una oportunidad para retomar su vida y comenzar de nuevo.

El presente estudio se llevó a cabo con víctimas residentes en la Urbanización Villas de San Pablo, en la periferia de Barranquilla. Este lugar es el "primer macroproyecto de vivienda nacional, focaliza sus esfuerzos en generar capital económico, social y ambiental, y crear comunidades autónomas y sostenibles" (Valencia, 2017, p. 65). Sus beneficiarios son familias en condiciones de vulnerabilidad, entre cuyos integrantes se encuentra un gran número de personas desplazadas por la violencia sociopolítica. El objetivo del estudio fue identificar los recursos psicosociales, y el empleo de los mismos, en víctimas con TEPT para el posterior diseño de un programa de intervención psicosocial (adecuado a la realidad analizada) que promueva su salud mental, $y$, además, aportar al conocimiento científico para el beneficio de nuevos estudios y programas de intervención.

\section{Evidencia de salud mental en víctimas de violencia sociopo- lítica}

Amarís, Blanco, Madariaga, Acuña, Valle, Giraldo y Artuz (2016), sistematizaron investigaciones cuyo eje temático fue: bienestar y trauma psicosocial en víctimas de violencia política. Estos autores revisaron estudios efectuados sobre en el bienestar psicológico, bienestar subjetivo y bienestar social, como indicadores de salud mental, relacionados con el Modelo del Estado Completo de Salud (MECS) (Keyes, 2005), el cual será explicado en el siguiente apartado. La integración de estas investigaciones fue de tipo cuantitativo y cualitativo, posibilitando "una reflexión teórica que mediante la comparación de realidades permitió evidenciar aspectos comunes en torno a los resultados empíricos de estudios" (Amarís et al., 2016, p. 216). Dentro de estos aspectos comunes se observan y describen estrategias de afrontamiento encontradas en las víctimas. Todo esto contribuyó a la elaboración de un perfil de salud mental basados en las características de los participantes de los estudios revisados.

El perfil permitió obtener evidencias de bienestar como indicadores de salud en los individuos. A pesar de la experiencia traumática y los efectos de la misma sobre la salud, la pobreza en la que se hallan por causa del desplazamiento, el quebrantamiento en los lazos familiares y/o de amistades cercanas, las víctimas tienen la capacidad de emplear "estrategias adaptativas que les han permitido sobrevivir a las situaciones de sufrimiento y encontrarle sentido a la existencia" (p. 217). 
Algunos de los indicadores de bienestar que hallaron y que fueron expuestos dentro de su investigación son: "1) el reconocimiento del amor por sus hijos; 2) el sentir que están frente a una nueva oportunidad en la vida, 3) el reconocer que tienen habilidades y competencias que les permite salir adelante" $p$. 217). Se suma a estos el apoyo social de vecinos y familiares, al igual que la confianza en sí mismos y en Dios. Igualmente, la presencia de objetivos de vida, relacionados a su futuro y el de sus hijos, fueron enmarcados por los autores dentro de la dimensión de propósito de vida.

\section{Salud mental: Estado de bienestar}

De acuerdo a la Organización Mundial de la Salud - OMS (2013), la salud mental es un estado de completo bienestar físico, mental, social y no sólo ausencia de enfermedad. Es un estado de bienestar donde existe consciencia de las propias capacidades; se afronta las situaciones estresantes del diario vivir, se actúa de modo productivo y con capacidad de aportar al entorno.

Keyes (2002), dentro de su definición, también destaca al bienestar como eje fundamental de la salud mental. Éste la describe como un "conjunto de síntomas de hedonía y funcionamiento positivo operacionalizado por medidas de bienestar subjetivo - percepciones individuales y evaluaciones que se hace sobre la propia vida y sobre la calidad de su funcionamiento" (Keyes 2002, p. 540). Desde las definiciones presentadas, se observa una estrecha relación entre la salud mental y el bienestar, donde se destaca un estado positivo del individuo y su capacidad para valorar el diario vivir.

Keyes (2005), planteó el MECS, como medida de salud mental basado en las dimensiones del bienestar: subjetivo, psicológico y social. Blanco y Blanco (2018), quienes han basado sus investigaciones con población desplazada sobre el MECS, explican brevemente a cada uno de ellos: el bienestar subjetivo comprende las experiencias emocionales, el sentimiento de satisfacción, al igual que el juicio global de los individuos sobre el desarrollo de su vida en relación con el sentimiento de satisfacción o insatisfacción. El bienestar psicológico, "pone el énfasis en el crecimiento personal, en el desarrollo del potencial humano" (p. 133). Mientras que el bienestar social atañe a la salud en su relación con el contexto social. En palabras de los autores, es:

"la valoración que hacemos de las circunstancias y el funcionamiento dentro de la sociedad" (Keyes, 1998, p. 122), que nos permiten sentirnos integrados, confiar en los otros y en la sociedad en la que nos ha tocado vivir, percibir que somos de alguna utilidad y que se nos tiene en consideración, que se nos valora y se nos atiende había dicho Ryff. (Blanco y Blanco, 2018, p. 126).

Díaz, Blanco, Horcajo y Valle (2007), declaran la existencia de una exhaustiva investigación realizada en los últimos cuarenta años sobre el bienestar, en la cual resaltan que la propuesta de Corey Keyes es una de las más influyentes. Esta propuesta (el MECS), permite ver dos aspectos: "Primero: más que formar una única dimensión bipolar, la salud y la enfermedad son dos dimensiones unipolares correlacionadas entre sí. Segundo: la presencia de salud mental supone la existencia de un funcionamiento psicosocial positivo" (p.286).
La presente investigación empleó indicadores cualitativos basados en las dimensiones del MECS, que permitieron la construcción del perfil de salud mental de las víctimas. Se aclara, que los autores de este trabajo tomaron al MECS como marco de referencia, considerando que podría dar cuenta de los efectos de la violencia en las víctimas, en este caso, las secuelas del desplazamiento forzado sobre la salud mental.

El empleo de recursos: Estilos y estrategias de afrontamiento La salud mental y el afrontamiento presentan una estrecha relación, ya que éste último "forma parte de los recursos psicológicos o psicosociales que el individuo utiliza para hacer frente a situaciones estresantes" (Amarís, Madariaga, Valle y Zambrano, 2013, p. 128); comportándose como una variable moderadora que regula los efectos desestabilizadores del estrés (Kirchner, 2003). Por lo tanto, se entiende, que de acuerdo al modo en que se emplee el afrontamiento, éste afectará (de una u otra forma) en el bienestar de los individuos. Por ello, el perfil elaborado aquí, también se relaciona en gran parte con el modo en que los participantes del estudio emplean sus recursos psicosociales, es decir, con sus estilos y estrategias de afrontamiento.

Dentro de las teorías del afrontamiento, como las de Lazarus y Folkman (1986), o las de Carver, Scheier \& Weintraub (1989), se evidencia la presencia de estilos activos y pasivos (Krzemien, Monchietti y Urquijo, 2005; Buceta, Bueno y Mas, 2001). Los activos son considerados positivos, pues, permiten una adaptación satisfactoria (Krzemien, Monchietti y Urquijo, 2005); mientras que los pasivos, son vistos de una forma negativa, pues, dificultan el afrontamiento adecuado, incrementando la vulnerabilidad hacia el estrés (Buceta, Bueno y Mas, 2001). En el contexto del desplazamiento, las investigaciones indican que las estrategias de afrontamiento adecuadas promueven el fortalecimiento de la salud mental en las víctimas (Amarís et al., 2016).

En resumen, el perfil de salud mental de este estudio, surge de la exploración cualitativa donde se integran las tres tradiciones del bienestar, así como los recursos psicosociales de los participantes del estudio y sus formas de emplearlos, revelando estrategias de afrontamiento que denotan estilos activos y pasivos que conforman su perfil de salud mental.

\section{Diseño}

\section{Metodología}

Esta investigación, de corte cualitativo, contó con una exploración a partir de un estudio de caso colectivo; aquí se efectuó un taller investigativo y entrevistas en profundidad con víctimas que fueron previamente diagnosticadas con TEPT, como criterio de inclusión para el ingreso a la etapa de exploración.

\section{Participantes}

El enlace con la población fue posible gracias la Oficina de Desarrollo Integral de Comunidades Sostenibles (DINCS) de la Fundación Mario Santo Domingo y a líderes comunitarias de la urbanización. Los participantes fueron hombres y mujeres mayores de edad, habitantes de la urbanización Villas de San Pablo en la ciudad de Barranquilla, reconocidas como víctimas del desplazamiento forzado dentro del Registro Único de Víctimas (RUV), quienes presentaban un diagnóstico previo de TEPT. El diagnóstico fue un primer paso para la identificación de los individuos, y de selección para la exploración. El muestreo fue de tipo intencional, bajo convocatoria. 
Posteriormente, en la fase de exploración, se contó con la participación voluntaria de 32 personas, quienes fueron informados de los objetivos, confidencialidad de la información y alcances de la investigación. Estos sujetos firmaron el consentimiento informado para esta etapa. A partir del criterio de saturación de esta muestra, se obtuvieron las categorías esperadas a encontrar dentro del análisis, correspondiendo tanto con los objetivos de la investigación como con los hallazgos en la literatura del desplazamiento forzado. Así mismo, se entiende que esta muestra no tiene por objeto la representatividad estadística, sino la intención de captar significados, pistas, explicaciones, sentimientos, conductas, opiniones, etc, que aporten sobre la comprensión del fenómeno de estudio (Ruiz, 2015).

\section{Instrumentos}

Para la recolección de información se realizó un taller investigativo y entrevistas en profundidad. Además, las definiciones de las dimensiones de las escalas de Bienestar subjetivo de Diener, psicológico de Ryff, y social de Keyes, así como de las estrategias de afrontamiento de las escalas de Coping Modificada (EEC-M), fueron tomadas como referentes para la creación de indicadores cualitativos, los cuales permitieron, mediante la observación, el registro de evidencias de bienestar y estrategias de afrontamiento empleados por los sujetos, y cuya información también fue objeto de análisis. Estas se usaron como base para la creación de los indicadores, teniendo en cuenta que serán empleadas en la segunda fase de la investigación que consta del diseño, implementación y evaluación de un programa de intervención psicosocial para las víctimas.

Cabe agregar, que los indicadores cualitativos, se refieren aquí, a aspectos, significados o percepciones sociales y no a cantidades (Alonso, 1998), que sirvieron como pautas de observación, dando un mayor sentido a los datos recolectados, basados en la interacción social generada con los participantes para el enriquecimiento de la investigación.

\section{Taller investigativo}

El taller investigativo, es en sí mismo, un instrumento social intencionado, con él se quiere obtener información con un propósito. Es decir, se enmarca dentro de unos objetivos y metas donde se busca construir conocimiento. La construcción de conocimiento, le permite ser flexibles y cambiante (Ghiso, 1999).

En el taller investigativo, de la presente investigación, se realizaron cinco actividades para abordar la relación entre la condición como desplazado y las diferentes dimensiones sociales (familiar, institucional, comunitaria y laboral). Se discutieron, por grupos al azar, las experiencias vividas sobre dichas dimensiones, identificando dificultades y posibles soluciones. Así mismo, los participantes realizaron lluvias de ideas sobre problemáticas y soluciones, las cuales fueron registradas en carteleras y representadas luego en un role play, intentando comprender sus experiencias y sentimientos, como un modo de acercamiento al contexto en el que se desarrollan. En otra actividad, se buscó conocer y comprender sus perspectivas de vida antes y después del desplazamiento; aquí se evidenciaron diferencias en sus relaciones sociales, sentimientos, emociones y percepción de sí mismos. Igualmente, se abarcó la dimensión del bienestar, a partir del significado que éste tiene para ellos, cómo lo buscan y cómo afrontan la adversidad.
Por último, se creó un espacio para comentarios y conclusiones para validar la información recolectada. Los registros del taller fueron efectuados mediante filmación (los participantes dieron su consentimiento para ser grabados); registro de los indicadores cualitativos y las notas de campo.

\section{Indicadores cualitativos}

Estos se basaron en las dimensiones descritas sobre el Bienestar psicológico (Blanco y Díaz, 2005), Bienestar social (Díaz, Rodríguez-Carvajal, Blanco, Moreno-Jiménez, Gallardo, Valle y Dierendonck, 2006), ítems del bienestar subjetivo (Diener, Emmons, Larsen \& Griffin, 1985), y las estrategias de afrontamiento descritas por Londoño et al. (2006), los cuales son evaluados en las escalas propuestas/adaptadas por dichos autores. Estos sirvieron como pautas de observación.

\section{Entrevistas en profundidad}

La entrevista en profundidad permite comprender la realidad y aproximarse a ella por medio de la interacción entre el entrevistado y el entrevistador, logrando la inmersión del entrevistador en una posición de observador dentro del mundo social que tiene como objeto de estudio. El contenido del discurso se va orientando por medio de la exploración dentro de un determinado contexto cultural que permiten comprender su significado (Ruiz, 2015).

En este estudio, se realizaron entrevistas en profundidad semiestructuradas a cuatro personas, las cuales mostraron mayor presencia de síntomas de TEPT de acuerdo a los hallazgos en las convocatorias y a lo observado en el taller investigativo. Se entrevistaron sólo a cuatro personas teniendo en cuenta que el objetivo de esta "no obedece a una representación estadística, sino que consiste en el estudio minucioso de la información que se obtenga de las conversaciones con los entrevistados" (Robles, 2011, p. 42). Los registros de las entrevistas se realizaron mediante la grabación de voz, autorizada por los participantes.

\section{Procesamiento de la información}

Los registros obtenidos de las diferentes técnicas (taller investigativo, entrevista en profundidad), así como de los indicadores cualitativos, permitieron la triangulación de la información, los cuales fueron sometidos a un análisis de contenido de tipo cualitativo, enmarcado desde el enfoque fenomenológico-social, donde se estudiaron varios casos para comprobar la generalidad del fenómeno. Se conformaron las subcategorías y posteriormente las categorías como resultados de la exploración. Para el procesamiento se empleó el software Atlas.ti en su versión 8.0.

\section{Resultados}

Se obtuvieron seis categorías de análisis, denominadas categorías de referencia inicial. Este nombre se debe a que, si bien las categorías se elaboraron a partir del análisis de contenido, ya se tenían ideas a priori de los factores o aspectos a encontrar, como consecuencia de la interacción con los participantes durante las actividades exploratorias, y la revisión de la literatura referida a víctimas de violencia sociopolítica. El análisis de contenido ayudó a dar cuerpo a las ideas y determinar las categorías.

\section{Categorías de referencia iniciales}

De acuerdo a Lazarus y Folkman, existen variables que influ- 
yen en las estrategias de afrontamiento usadas por los individuos en situaciones que generan estrés; dichas variables son recursos que influyen en el afrontamiento, y pueden ser: la salud, la energía física, las creencias existenciales/espirituales, las técnicas de resolución de conflictos, las habilidades sociales, las creencias del control del individuo sobre sí mismo y/o su medio, los recursos materiales, entre otros (Di-Colloredo, Aparicio y Moreno, 2007). Autores como Amarís et al. (2016) y Abello, Amarís, Blanco, Madariaga, Manrique, Martínez \& Díaz (2009), exponen la evidencia de recursos psicosociales como indicadores de salud mental en víctimas, basándose en el MECS.

Las categorías conformadas en esta investigación son consideradas recursos psicosociales con los que cuentan los participantes, las cuales en su nombre reflejan características positivas o negativas, apoyadas por el contenido de cada subcategoría que las conforma (Ver tabla 1). Esto posibilitó que las mismas fueran englobadas dentro de dos factores: protectores o de riesgo.

Se entiende en esta investigación, que los factores protectores son características, ya sean propias de individuos, grupos o comunidades, o características externas (entorno/situación), que permiten reducir la probabilidad de que se dé un comportamiento desviado (Pérez \& Mejía, 1998, citados en Villanueva-Bonilla y Ríos-Gallardo, 2018), o algún tipo de enfermedad (Villanueva-Bonilla y Ríos-Gallardo, 2018; Muñoz, Sandstede, Klimenko 2017); o trastorno mental (Corona, Hernández y García, 2016), disminuyendo la vulnerabilidad o las amenazas de manera que contrarresta los efectos de los factores de riesgo, favoreciendo el desarrollo humano (Páramo, 2011).
Por su parte, se consideran factores de riesgo a las características (internas o externas del sujeto, grupo o comunidad) que incrementan la probabilidad de producir alguna enfermedad (Villanueva-Bonilla y Ríos-Gallardo, 2018), o un determinado fenómeno (Luengo et al., 1999, citado en Muñoz, Sandstede, y Klimenko (2017), o la vulnerabilidad (Corona, Hernández y García, 2016), hacia comportamientos indeseados, impactando negativamente en el (los) individuo(s).

Las características de las categorías, que conformaron los factores de protección y de riesgo en este estudio, coincidieron con los hallazgos en la literatura del desplazamiento forzado, donde se presentaban tanto posturas fatalistas como activas en las víctimas. Las posturas fatalistas se definen como "la ausencia de un panorama claro de posibilidades (...) lo que le da apertura a una postura fatalista ante el futuro" (Amarís et al., 2016, p. 219). Mientras que las posturas activas se evidencian en la "movilización ante las necesidades diarias, desde el rebusque para encontrar recursos económicos, hasta la gestión de papeles para tomar las ayudas institucionales" (Amarís et al., 2016, p. 219) Estos factores también se relacionan con los estilos activos y pasivos del afrontamiento.

En resumen, las categorías obtenidas, exponen los recursos psicosociales positivos y negativos empleados por los participantes, los cuales coinciden con la literatura del desplazamiento forzado. En la tabla 1 se presentan las categorías y sus correspondientes subcategorías que llevaron a configurarlas dentro de los factores protectores o de riesgo.

Tabla 1. Identificación de las subcategorías encontradas en el análisis de contenido.

\begin{tabular}{|c|c|c|c|c|}
\hline & Nombre de las Categorías & CódC & Nombre de las Subcategorías & CódS. \\
\hline \multirow{8}{*}{ Factores Protectores } & \multirow{2}{*}{ Tener un propósito de vida } & \multirow{2}{*}{1} & Hijos como motor de vida & 1.1 \\
\hline & & & Deseos de cumplir metas y superarse & 1.2 \\
\hline & \multirow{2}{*}{ Apoyo espiritual } & \multirow{2}{*}{2} & Fortaleza en creencias espirituales & 2.1 \\
\hline & & & Agradecimiento por las bendiciones recibidas & 2.2 \\
\hline & \multirow{4}{*}{ Reevaluación positiva } & \multirow{4}{*}{3} & Vecinos como red de apoyo & 3.1 \\
\hline & & & Interés en recibir apoyo psicológico & 3.2 \\
\hline & & & Cambios conductuales positivos a raíz del desplazamiento & 3.3 \\
\hline & & & Conductas de distracción como formas de afrontamiento & 3.4 \\
\hline \multirow{11}{*}{ Factores de Riesgo } & \multirow{2}{*}{$\begin{array}{l}\text { Dificultad en la } \\
\text { auto-aceptación }\end{array}$} & \multirow[t]{2}{*}{4} & Subvaloración & 4.1 \\
\hline & & & Sentimientos/conductas de aislamiento social & 4.2 \\
\hline & \multirow{7}{*}{$\begin{array}{l}\text { Sentimientos de poco } \\
\text { dominio sobre el entorno }\end{array}$} & \multirow[t]{7}{*}{5} & Desesperanza por sufrimiento pasado y presente & 5.1 \\
\hline & & & Revictimización por parte del Estado & 5.2 \\
\hline & & & Tristeza generada por el desplazamiento. & 5.3 \\
\hline & & & Desconfianza & 5.4 \\
\hline & & & Discriminación & 5.5 \\
\hline & & & Falta de apoyo/conflicto familiar & 5.6 \\
\hline & & & Carga emocional/trabajo duro & 5.7 \\
\hline & \multirow{2}{*}{$\begin{array}{l}\text { Conductas agresivas no } \\
\text { adaptativas }\end{array}$} & \multirow{2}{*}{6} & $\begin{array}{l}\text { Cambios conductuales negativos } \\
\text { a raíz del desplazamiento }\end{array}$ & 6.1 \\
\hline & & & $\begin{array}{l}\text { Problemas de convivencia/ } \\
\text { revictimización comunitaria }\end{array}$ & 6.2 \\
\hline
\end{tabular}

Fuente: Elaboración propia. 


\section{Factores protectores}

\section{Tener un propósito de vida}

Son los propósitos y objetivos manifestados por los participantes, aquellos que los han impulsado a dar sentido a su vida y seguir adelante. Lo conforman dos subcategorías:

Hijos como motor de vida: Durante la exploración se evidenció que los participantes perciben a sus hijos como la fuerza que los impulsa a salir adelante. A su vez, ellos quieren que sus hijos logren entrar a la universidad para superar las dificultades de la vida; aquellos que ya tienen hijos universitarios o cursando estudios, sienten orgulloso de esto.

Deseos de cumplir metas y superarse: Los participantes quieren validar el bachillerato, crear un negocio, pagar la educación superior de los hijos, estudiar una carrera de educación superior para dar ejemplo y lograr una mejor calidad de vida, cambiar la perspectiva de vida y superar su pasado negativo.

\section{Apoyo espiritual}

Esta categoría surgió porque los participantes reiteraron, en su discurso, la figura de Dios, a quien ven como protector y fuente de fortaleza. Aseguran tener fe y agradecimiento hacia él. De esta categoría se desprenden dos subcategorías:

Fortaleza en creencias espirituales: Los participantes sostienen la existencia de un ser supremo que los acompaña, protege y escucha sus plegarias. Su fe en Dios les da fortaleza para sobrellevar los problemas de la vida diaria y tratar de superar traumas como el desplazamiento o la muerte de seres queridos por cuenta de la violencia. Agradecimiento por las bendiciones recibidas: LoS participantes afirman que fueron bendecidos por Dios cuando recibieron su casa en Villas de San Pablo. También le agradecen por su salud, el dinero que reciben para comprar comida, y la vida que llevan alejada de la violencia de sus lugares de origen.

La interpretación del discurso sugiere que creer en un ser supremo ayuda a soportar traumas del pasado y situaciones adversas actuales. Esto sucede porque se experimenta la sensación de un apoyo superior y la esperanza de soluciones futuras. Por esta razón, el apoyo espiritual es considerado un factor protector.

\section{Reevaluación positiva}

Está relacionada con las actitudes o disposiciones positivas para afrontar los problemas, ya sean a generados a partir del desplazamiento, o en el momento presente. Igualmente, la identificación de las herramientas para salir adelante y los recursos cognitivos propios de los participantes, hacen parte de la conceptualización de esta categoría. Las subcategorías que conforman la reevaluación positiva y que se caracterizan como factores protectores, son:

Vecinos como red de apoyo: Los participantes consideran que conversar con los vecinos los distrae frente a sus problemas; así mismo, recibir ayuda material como alimentos o consejos familiares los anima y fortalece.

Interés en recibir apoyo psicológico: Los participantes manifestaron necesitar una ayuda u orientación psicológica para tener herramientas intrínsecas y extrínsecas que les permita enfrentar las dificultades de la vida diaria, al igual que tomar decisiones que ayuden a resolver conflictos en su comunidad y en la familia, especialmente con sus hijos.

Cambios conductuales positivos a raíz del desplazamiento: A pesar de las consecuencias que trajo el desplazamiento, los individuos sienten que ahora son más fuertes ante los problemas; algunos aseguran estar más unidos con sus familias y ser más sociables con los demás.

Conductas de distracción como formas de afrontamiento: Los participantes, cuando se sienten tristes o experimentan eventos negativos, echan mano de comportamientos para sentirse mejor, como salir a la calle, conversar con vecinos, tomar una ducha, tejer, ver televisión, leer, aprender cosas nuevas por internet o leer la Biblia.

\section{Factores de riesgo}

\section{Dificultad en la auto-aceptación}

Se refiere a las dificultades de los participantes para resaltar sus fortalezas y cualidades internas. Se observó una subestimación de la propia capacidad intelectual relacionada al nivel educativo. En otras palabras, el hecho de que la mayoría no haya culminado la secundaria o adelantado estudios posteriores, los intimida ante el mundo laboral; por esta razón, se sienten discriminados al momento de buscar trabajo. Los recuerdos del desplazamiento y los tantos problemas del presente hacen que se sientan solos y/o se aíslen como una forma de preservar bienestar; en consecuencia, limitan su relación con los demás, lo que puede afectar la concepción positiva sobre sí mismo. Las subcategorías que conforman son:

Subvaloración: Los participantes manifiestan no contar con la inteligencia suficiente para conseguir una vida exitosa o un buen trabajo, debido a que no terminaron la primaria o el bachillerato. Algunos incluso se definen a sí mismos como brutos por su origen campesino, y sienten que las personas se burlan de ellos por su educación, sus características culturales o por el hecho de ser desplazados. Por otro lado, se sienten mal por no tener el bienestar que les ofrecía la vida en el campo, mostrándose decepcionados por lo que han logrado ahora.

Sentimientos/conductas de aislamiento social: Los participantes dicen sentirse solos debido a los problemas familiares y comunitarios que enfrentan. El pasado traumático y el difícil presente los has vuelto desconfiados. Estos sentimientos de soledad y/o aislamiento se relacionan mayormente con la percepción de contar con pocas herramientas intrínsecas para resolver la adversidad, lo que afecta su autoestima. Por ello, aunque puede ser relacionada a la categoría de sentimientos de poco dominio sobre el entorno, se considera más un aspecto de la autoestima que debe ser orientado para un mejor manejo del sentimiento y las conductas solitarias, pues se cree que, si se fortalece la autoestima, el sentimiento de soledad y las conductas de aislamiento disminuirán.

\section{Sentimientos de poco dominio sobre el entorno}

Los participantes, en general, sienten que no tienen control sobre los acontecimientos externos. En otras palabras, creen que su poder sobre las situaciones adversas es mínima o nula. Dejan todo en mano de Dios, creyendo que sólo un poder superior cambiará las cosas. Las subcategorías conformadas son: 
1) Desesperanza por sufrimiento pasado y presente: Estos Individuos se sienten desalentados, pesimistas sobre su porvenir relacionado a lo que han vivido y siguen viviendo luego del desplazamiento. La desesperanza se relaciona también con la muerte o asesinato de parientes durante y luego del desplazamiento, lo que hace que tengan pensamientos negativos sobre la vida en general. Entre las manifestaciones de desesperanza se encuentran: limitantes para obtener trabajo por su edad, pérdidas bienes, tener un empleo no deseado, los múltiples desplazamientos sufridos (en algunos casos), la pobreza en la que siguen viviendo, las enfermedades y/o infartos padecidos por ellos o por sus familiares luego del desplazamiento, problemas familiares y de pareja, sentimiento de persecución y metas frustradas.

Aunque indican que tratan de superarse, los afecta en gran medida las problemáticas comunitarias, y el sentimiento de desamparo por parte del Estado.

2) Revictimización por parte del Estado: Los participantes manifiestan que las instituciones estatales están poco comprometidas con ellos, aunque reconocen haber recibido algunos beneficios luego de varias luchas, ya que los procedimientos legales son lentos y dispendiosos. Aseguran que es difícil acceder al sistema educativo, en especial a la educación superior. Esta sensación de desamparo los revictimiza.

3) Tristeza generada por el desplazamiento: Añorar el lugar de origen, la muerte de seres queridos, decepción sobre su vida actual, la pobreza, ejercer trabajos diferentes a los del campo, la falta de apoyo por parte de la familia y/o del estado, problemas de pareja y comunitarios y la soledad, generan estos sentimientos de tristeza.

4) Desconfianza: Se relaciona la desconfianza en mayor parte con la comunidad en la que se vive, pues existe los roces entre vecinos, conductas de aislamiento, conductas agresivas, diferencias culturales, drogadicción, robos, etc. Se considera, que por estas razones, las conductas son más defensivas y agresivas entre las personas de la comunidad.

5) Discriminación: Los participantes han tenido diversos tipos de experiencias discriminatorias: intolerancia por parte de la comunidad por su lugar de origen y acento, rechazo por su color de piel, discriminación social, y limitantes para conseguir empleo por su condición de desplazado o su nivel de estudios.

6) Falta de apoyo/conflicto familiar: No se sienten apoyados por sus parejas; consideran que hay poca comunicación y falta de apoyo económico por parte de sus familiares, sean o no de su núcleo familiar. A esto se suman los problemas con los hijos y sus exigencias sobre mejores condiciones de vida. Por estas razones se evidencia una carga emocional que recae en ellos, y que también los hace sentir desamparados.

7) Carga emocional/trabajo duro: Después del desplazamiento, los participantes han sentido una mayor carga emocional. Aun así, están dispuestos a realizar trabajos duros y sacrificios para asegurar su bienestar: cuidan a sus padres (en algunos casos enfermos), trabajan sin descanso, procuran obtener beneficios por ser desplazados, tienen dificultades para dormir debido a los problemas económicos y animan a sus parejas a seguir adelante, aunque sienten que no les colaboran.

\section{Conductas agresivas no adaptativas}

Esta categoría evidencia que los participantes presentan conductas agresivas e impulsivas ante la sociedad en general, que, aunque pueden ser vistas como comportamientos defensivos ante las vivencias del día a día, y que están relacio- nadas con el trauma del desplazamiento, tienden a ser como lo denomina, Fromm (1975), conductas agresivas malignas, o no adaptativas, puesto que se sobrepasan los límites, transgrediendo fuertemente tanto verbal como físicamente. En algunos casos existen amenazas de muerte con la muestra explícita de armas, o golpes entre vecinos por irrumpir la tranquilidad del otro (por ejemplo, colocar música en alto volumen y en horas no adecuadas), mostrando intolerancia y desinterés por el respeto al espacio vital de los demás.

Se observa, además, dificultad para conciliar conflictos que ayuden a mejorar la convivencia comunitaria. Se considera que las víctimas reaccionan agresivamente, pues, han vivido dentro de la violencia y la han reproducido en su vida diaria, haciendo que estos comportamientos formen parte de sus mecanismos de defensa. Las subcategorías que la componen son:

1) Cambios conductuales negativos a raíz del desplazamiento: Reportaron que luego del desplazamiento, se tornaron muy agresivos. Se sienten abandonados por el estado y están permanentemente a la defensiva con la comunidad o la sociedad. Varios manifestaron haber cambiado su forma de ser, antes despreocupada, tranquila, alegre y relajada a ser más serios, estar más angustiados y temerosos, desconfiados y reaccionar más rudamente.

2) Problemas de convivencia/revictimización comunitaria: Para los participantes, la convivencia en sus comunidades es difícil por la falta de tolerancia derivada de las diferencias culturales. Hay agresiones físicas y verbales, amenazas de muerte y malos entendidos. Además, en la urbanización en que residen hay problemas de drogadicción y robos constantes, lo que los lleva a aislarse del entorno.

\section{Discusión}

Con base en los resultados, se permite establecer relaciones entre las categorías de referencia iniciales y la revisión realizada por Amarís et al. (2016). Estos autores indican que, en las posturas activas, los recursos psicosociales mayormente empleados por las víctimas son, "en primer lugar, el significado de los hijos, quienes son el motivo para salir a trabajar, buscar sentirse mejor e inclusive pensar en posibilidades futuras de progreso y estabilidad" (p. 219). En segundo lugar, la espiritualidad, es decir, la creencia en Dios como fuente de tranquilidad y agradecimiento, que sirve como apoyo o amortiguador del trauma y del estrés de la vida cotidiana. Estos recursos activos coinciden con las categorías conformadas en los factores protectores.

Además, en diversas investigaciones se evidencia que algunas víctimas de desplazamiento son capaces de valorar los recursos familiares e institucionales que pueden estar a su alcance, mostrándose abiertos y flexibles ante la búsqueda de soluciones, mostrando "habilidad para afrontar y resolver problemas del día a día (...) aunque implique el ensayo de diversos caminos hasta lograr hallar el adecuado" (Amarís et al., 2016, p. 224). Esto apunta a la búsqueda de soluciones y a la reevaluación positiva como estrategias de afrontamiento activo.

En cuanto a las dimensiones de autoaceptación y relaciones con los otros se ven afectadas, observándose la subvaloración, el debilitamiento de la autonomía y de la autoconfianza relacionada con el evento traumático, afectando en la identi- 
dad y la autoestima, generando incertidumbre, desesperanza en el desarrollo social y en las instituciones (Blanco y Amarís, 2014., y Amarís et al., 2009 citados en Amarís et al., 2016). A su vez, la tristeza, la desesperación y la añoranza de su vida pasada, acompañada por creencias sobre un incongruente funcionamiento social y la poca capacidad de cambiar el rumbo de las cosas, son posturas fatalistas (Amarís et al., 2016), que se asemejan con la categoría de Sentimiento de poco dominio sobre el entorno. Estos aspectos atañen a los factores de riesgo hallados en el presente estudio. Por lo tanto, se plantea que estas actitudes o posturas fatalistas son comunes entre las víctimas.

Teniendo en los resultados, se observa que las víctimas presentan un patrón común de recursos psicosociales que emplean para afrontar ante la adversidad. Estos recursos o estrategias pueden ser fatalistas, que se relacionan con los estilos pasivos/ evitativos del afrontamiento. $Y$, por otro lado, las posturas activas que demuestran estilos activos de afrontamiento como indicadores de salud mental.

Autores como Hoyos (2014), y Domínguez y Godín (2007), proponen la creación de nuevos diseños reconociendo los recursos y/o factores protectores de los sujetos a partir de la investigación misma, lo cual contribuye a la desvictimización de los sujetos afectados al reconocerlos como agentes activos. Es decir, se propone manejar el tema del desplazamiento forzado desde una perspectiva positiva, estimulando a las víctimas al reconocimiento de sus habilidades que los ayude a afrontar activa y adecuadamente su realidad, oponiéndose a teorías victimizantes, ayudando a los individuos afectados a la apropiación de su transformación.

Con base en todo lo anterior, se puede afirmar que la exploración permitió una aproximación sobre la salud mental y el empleo de recursos psicosociales por parte de víctimas del desplazamiento forzado. Esto contribuyó al conocimiento sobre las formas de afrontar las situaciones de su vida, aportando a la construcción de un programa de intervención psicosocial para el mejoramiento de la salud mental de estos individuos.

Teniendo en cuenta los resultados, se permite decir que los participantes evidenciaron un perfil de salud mental semejante a lo encontrado en la literatura de víctimas de violencia sociopolítica, con uso de recursos psicosociales similares para afrontar la adversidad. Estos recursos o estrategias pueden ser negativos/fatalistas o positivos/activos, denominados en la investigación como factores de riesgo y factores protectores, algo que se considera importante para la configuración del programa de intervención. Las categorías derivadas del análisis ayudaron a identificar los recursos psicosociales más empleados por los participantes, así como el impacto del desplazamiento en su vida.

Por último, en el campo del desplazamiento forzado, no se observa suficiente información relacionada a estrategias de salud mental en víctimas que sirva de aporte a nuevos programas de intervención, por lo que se considera necesario efectuar más estudios, programas y publicaciones en este aspecto. Se espera, con esta investigación, aportar al conocimiento científico para beneficio de nuevos estudios y programas de intervención. También, se propone el diseño e implementación de programas de intervención psicosocial, mediante la promoción de factores protectores y de recursos psicosocia- les, que se adecuen a la realidad de los individuos.

\section{Referencias}

Abello, R., Amarís, M., Blanco, A., Madariaga, C., Manrique, P., Martínez, M. \& Díaz, D. (2009). Bienestar y trauma en personas adultas desplazadas por la violencia política. Universitas Psychologica, 8(2), 455-470. Recuperado de: http://www.redalyc.org/articulo. oa?id=64712165014

Aguilar, M., Morillas, D., y Patró, R. (2011). Victimología. Un estudio sobre la víctima y los procesos de victimización. Madrid: Editor: Dykinson.

Alonso, L. E. (1998). La mirada cualitativa en sociología. Una aproximación interpretativa. Editorial: Fundamentos.

Amarís, M., Blanco, A., Madariaga, C., Acuña, E., Valle, M., Giraldo, V. y Artuz, L. (2016). Estrategias de intervención psicosocial dirigidas a la promoción y fortalecimiento de la salud mental en población víctima de la violencia política. En M. Gutiérrez y B. Sarmiento (Ed.), Gobierno y gobernanza: problemas, representaciones y políticas frente a graves violaciones a los derechos humanos (pp. 211-236). Cátedra UNESCO. Bogotá, D .C: Universidad Externado de Colombia.

Amarís, M., Madariaga, C., Valle, M., y Zambrano, J. (2013). Estrategias de afrontamiento individual y familiar frente a situaciones de estrés psicológico. Psicología desde el Caribe, 30(1), 123-145. Recuperado de: http://www.redalyc.org/articulo. oa?id=21328600007

Amarís, M., Abello, R., Acuña, E., Buelvas, F., Santamaría, A., y Turizo, Y. (2009). Construcción de estrategias de intervención psicosocial para la población desplazada y mujeres víctimas de violencia de género. Informe de Investigación, Departamento de Investigación y Desarrollo. Barranquilla: Universidad del Norte.

American Psychiatric Association., Kupfer, D. J., Regier, D. A., Arango López, C., Ayuso-Mateos, J. L., Vieta Pascual, E., \& Bagney Lifante, A. (2014). DSM-5: Manual diagnóstico y estadístico de los trastornos mentales (5a ed.). Madrid [etc.]: Editorial Médica Panamericana.

Arias, B. (2013). Salud mental y violencia política. Atender al enfermo psiquiátrico o reconocer al sujeto de la micropolítica. Revista Colombiana de Psiquiatría, 42(3), 276-282. doi: http://doi.org/10.1016/S00347450(13)70020-9

Blanco, R., y Blanco, A. (2018). La salud como un estado de bienestar. En: M. Amarís y C. Madariaga (Comp.), Intervenir para reparar: Recuperación de la dignidad y la salud mental en contextos de violencia (pp. 81-138). Barranquilla, Colombia: Universidad del Norte.

Blanco, A., y Amarís, M. (2014). La ruta psicosocial del desplazamiento: una perspectiva de género. Universitas Psychologica, 13 (2), 15-33. doi: 10.11144/Javeriana. UPSY13-2rpdu

Blanco, A., y Díaz, D. (2005). El bienestar social: Su concepto y medición. Psicothema, 17(4), 582-589. Recuperado de: https://www.redalyc.org/articulo. oa? id $=72717407$ 
Borda Bohigas, J. P., Carrillo, J. O., Garzón, D. F., Ramírez, M. P., y Rodríguez, N. (2015). Trauma histórico. Revisión sistemática de un abordaje diferente al conflicto armado. Revista Colombiana de Psiquiatría, 44(1), 41-49. doi: https://doi.org/10.1016/j.rcp.2014.09.005

Buceta, J., Bueno, A., y Mas, B. (Eds) (2001). Intervención psicológica y salud: control del estrés y conductas de riesgo. Madrid: Editorial Dykinson.

Campo-Arias, A., y Herazo, E. (2014). Estigma y salud mental en personas víctimas del conflicto armado interno colombiano en situación de desplazamiento forzado. Revista Colombiana de Psiquiatría, 43(4), 212217. doi: http://doi.org/10.1016/j.rcp.2014.09.004

Campo-Arias, A., Oviedo, H., y Herazo, E.(2014). Prevalencia de síntomas, posibles casos y trastornos mentales en víctimas del conflicto armado interno en situación de desplazamiento en Colombia: una revisión sistemática. Revista Colombiana de Psiquiatría, 43(4), 177-185. doi: http://doi.org/10.1016/j. rcp.2014.07.003

Carver, C.S., Scheier, M.F., \& Weintraub, J.K. (1989). Assessing coping strategies: Atheoretically based approach. Journal of Personality and Social Psychology, 56 (2), 267-283. doi: http://dx.doi.org/10.1037/00223514.56.2.267

Castaño, G., Sierra, G., Sánchez, D., Torres, Y., Salas, C., y Buitrago, C. (2018) Trastornos mentales y consumo de drogas en la población víctima del conflicto armado en tres ciudades de Colombia. Biomédica, 38, 77-92. doi:https://doi.org/10.7705/biomedica. v38i0.3890

Corona, B., Hernández, M., y García, R. (2016) Mortalidad por suicidio, factores de riesgos y protectores. Revista Habanera de Ciencias Médicas, 15(1), 90-100. Recuperado de: http://www.redalyc.org/articulo. oa?id=180444213011

Díaz, D., Blanco, A., Horcajo, J., y Valle, C. (2007). La aplicación del modelo del estado completo de salud al estudio de la depresión. Psicothema, 19, 286-294. Recuperado de: http://www.redalyc.org/articulo. oa? id=72719217

Díaz, D., Rodríguez-Carvajal R., Blanco, A., Moreno-Jiménez, B., Gallardo, I., Valle, C., y Dierendonck, D. (2006). Adaptación española de las escalas de bienestar psicológico de Ryff. Psicothema, 8(3), 572-577. Recuperado de: http://www.redalyc.org/articulo. oa?id=72718337

Di-Colloredo, C., Aparicio, D., y Moreno, J. (2007). Descripción de los estilos de afrontamiento en hombres y mujeres ante la situación de desplazamiento. Psychologia. Avances de la disciplina, 1(2) 125-156. Recuperado de http://www.redalyc.org/articulo. oa?id=297224996002

Diener, E., Emmons, R. A., Larsen, R. J., \& Griffin, S. (1985). The Satisfaction with Life Scale. Journal of Personality Assessment, 49, 71-75. doi: http://dx.doi. org/10.1207/s15327752jpa4901_13

Domínguez, E., y Godín, R. (2007). La resiliencia en familias desplazadas por la violencia sociopolítica ubicadas en Sincelejo. Psicología desde el Caribe, (19) 154-180. Recuperado de http://www.redalyc.org/ articulo.oa?id=21301907

Fromm, E. (1975). Anatomía de la destructividad humana. México: Siglo veintiuno editores.
Ghiso, A; (1999). Acercamientos: el taller en procesos de investigación interactivos. Estudios sobre las Culturas Contemporáneas, V(9), 141-153. Recuperado de http://www.redalyc.org/articulo.oa?id=31600907

Gómez-Restrepo, C., Tamayo-Martínez, N., Buitrago, G., Guarnizo-Herreño, C., Garzón-Orjuela., N., Eslava-Sch-

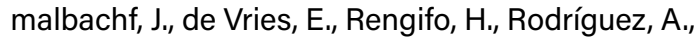
y Rincón, C.(2016) Violencia por conflicto armado y prevalencias de trastornos del afecto, ansiedad y problemas mentales en la población adulta colombiana. Violence due to armed conflict and prevalence of mood disorders, anxiety and mental problems in the Colombian adult population $R e$ vista Colombiana de Psiquiatría, 45, 147-153. doi: https://doi.org/10.1016/j.rcp.2016.11.001

Hewitt Ramírez, N., Juárez, F., Parada Baños, A J., Guerrero Luzardo, J., Romero Chávez, Y. M., Salgado Castilla, A. M., y Vargas Amaya, M. V. (2016). Afectaciones Psicológicas, Estrategias de Afrontamiento y Niveles de Resiliencia de Adultos Expuestos al Conflicto Armado en Colombia. Revista Colombiana de Psicología, 25(1) 125-140. Recuperado de http:// www.redalyc.org/articulo.oa?id=80444652009. doi: 10.15446/rcp.v25n1.49966

Hoyos, C. (2014). Hermenéutica de la resiliencia en víctimas de secuestro: en el marco de las nuevas concepciones restaurativas. Medellín, Colombia: Ediciones Unaula.

Keyes, C. (2005). Mental Illness and/or Mental Health? Investigating Axioms of the Complete State Model of Health. Journal of Consulting and Clinical Psychology, 73, 539-548. doi: 10.1037/0022-006X.73.3.539

Kirchner T. (2003). Estrategias de afrontamiento y nivel de psicoterapia en jóvenes presidiarios. Relación con el tiempo de reclusión y situación penitenciaria. $R e$ vista de Acción Psicológica, 2 (3), 199 - 211.

Krzemien, D., Monchietti, A., y Urquijo, S. (2005). Afrontamiento activo y adaptación al envejecimiento en mujeres de la ciudad de Mar del Plata: una revisión de la estrategia de autodistracción. Interdisciplinaria, 22 (2), 183-210. Recuperado de: http://www.redalyc.org/articulo.oa?id=18022204

Lazarus, R.S. \& Folkman, S. (1986). Estrés y procesos cognitivo [Stress and cognitive processes]. Barcelona: Martínez Roca.

Londoño, N., Henao, G., Puerta, I., Posada, S., Arango, D., y Aguirre-Acevedo, D. (2006). Propiedades psicométricas y validación de la Escala de Estrategias de Coping Modificada (EEC-M) en una muestra colombiana. Universitas Psychologica, 5 (2), 327349. Recuperado de http://www.redalyc.org/articulo.oa?id=64750210

Luengo, M. A., Tamames, R., Gómez, F., García, L. A., Pereiro, L. (1999). La prevención del consumo de drogas y la conducta antisocial en la escuela. Santiago de Compostela: Universidad de Santiago de Compostela.

Ministerio de Salud y Protección Social, Colciencias, Pontificia Universidad Javeriana, Datos, Procesos y Tecnología SAS. (2015). Encuesta Nacional de Salud Mental 2015. Tomo 1. Bogotá.

Muñoz, C., Sandstede, M., y Klimenko, O. (2017). Factores de riesgo y de protección para el consumo de drogas en los estudiantes del Instituto de Filosofía de la 
Universidad de Antioquia 2016. Psicoespacios, 11(18), 149-168. Recuperado de: http://revistas.iue. edu.co/index.php/Psicoespacios

Organización Mundial de la Salud -OMS (diciembre de 2013). Salud mental: un estado de bienestar. Recuperado de: https://www.who.int/features/factfiles/mental_health/es/

Páramo, M. D. L. A. (2011). Factores de Riesgo y Factores de Protección en la Adolescencia: Análisis de Contenido a través de Grupos de Discusión. Terapia psicológica, 29(1), 85-95. doi: http://dx.doi. org/10.4067/S0718-48082011000100009

Ramos-Vidal, I. (2018). Desplazamiento forzado y adaptación al contexto de destino: el caso de Barranquilla. Perfiles latinoamericanos, 26(51): 301-328. doi: http://dx.doi.org/10.18504/pl2651-012-2018

Robles, B (2011). La entrevista en profundidad: una técnica útil dentro del campo antropofísico. Cuicuilco, 18 (52), 39-49. Recuperado de: http://www.redalyc.org/ articulo.oa?id=35124304004

Ruiz, J. (2015). Las entrevistas en profundidad y la biografía. Revista San Gregorio. Metodología de la investigación, 1, 48-55. Recuperado de: http://revista.sangregorio.edu.ec/index.php/REVISTASANGREGO$\mathrm{RIO} /$ article/view/115

Shultz J.M., Gómez, A.M., Espinel, Z., Rios, S., Fonseca, M.F., \& Hernandez, L.J. (2014) Internal displacement in Colombia. Disaster Health, 2(1), 13-24. doi: 10.4161/ dish.27885

Silvera, L., y Amarís, M. (2017). Comprensión del bienestar desde las experiencias socioeconómicas de un grupo de mujeres desplazadas por la violencia sociopolítica en la ciudad de Barranquilla. Summa Psicológica, 14(1), 61-71. doi: 10.18774/summa-vol14. num1-319

Tamayo-Agudelo, W., \& Bell, V. (2018). Armed conflict and mental health in Colombia. BJPsych Internacional, 1-3. doi:10.1192/bji.2018.4

Tamayo Martínez, N., Rincón Rodríguez, C., De Santacruz, C., Bautista Bautista, N., Collazos, J., y Gómez-Restrepo, C. (2016). Problemas mentales, trastornos del afecto y de ansiedad en la población desplazada por la violencia en Colombia, resultados de la Encuesta Nacional de Salud Mental 2015. Revista Colombiana de Psiquiatría, 45 (1), 113-118. doi: http:// dx.doi.org/10.1016/j.rcp.2016.09.004

Valencia, L. (2017). Capítulo 2: Estudio de caso: Modelo de desarrollo integral de comunidades sostenibles de la Fundación Mario Santo Domingo en Villas de San Pablo, Barranquilla. En Romero, M. (Ed). Fundaciones, Inversión Social y Desarrollo (pp. 63125). Bogotá: Editorial Universidad Externado de Colombia.

Villanueva-Bonilla, C., y Ríos-Gallardo, Á. M. (2018). Factores protectores y de riesgo del trastorno de conducta y del trastorno de déficit de atención e hiperactividad. Una revisión sistemática [Protective and risk factors for conduct disorder and attention deficithyperactivity disorder. A systematic review]. Revista de Psicopatología y Psicología Clínica, 23(1), 59-74. doi: http://dx.doi.org/10.5944/rppc.vol.23. num.1.2018.19582 